\title{
Thomas Mormann
}

\section{TOPOLOGICAL ASPECTS OF COMBINATORIAL POSSIBILITY}

\begin{abstract}
The aim of this paper is to show that topology has a bearing on combinatorial theories of possibility. The approach developed in this article is "mapping account" considering combinatorial worlds as mappings from individuals to properties. Topological structures are used to define constraints on the mappings thereby characterizing the "really possible" combinations. The mapping approach avoids the well-known incompatibility problems. Moreover, it is compatible with atomistic as well as with non-atomistic ontologies. It helps to elucidate the positions of logical atomism and monism with the aid of topological separation axioms.
\end{abstract}

\section{CONTENTS}

1. Introduction, p. 76

2. Combinatorial possibility and world mappings, p. 77

3. Complex individuals and topological structures, p. 80

4. Topologically possible combinatorial worlds, p. 83

5. A topological elucidation of atomism and monism, p. 87

6. Concluding remarks, p. 90 


\section{Introduction}

The aim of this paper is to show that topology has a bearing on combinatorial theories of possibility. The basic idea of combinatorial theories of possibility is to conceptualize possible worlds as alternative combinations of entities populating the actual world. According to the approach of unrestricted combinatorialism the idea of possibility is to be be traced back to all combinations (cf. Armstrong (1989:37) whereas restricted combinatorialism considers it as the basic problem of a combinatorial approach to distinguish in a noncircular way between two sorts of combinations - the possible ones and the impossible ones (cf. Bigelow 1988).

The topological approach to be developed in this paper is a restricted combinatorialism, i.e., I consider it an essential task of a combinatorial theory to draw a line between possible and impossible combinations. More precisely, I am going to cast the combinatorial theory in the framework of a "mapping account" that considers combinatorial worlds as mappings from individuals to properties (cf. Skyrms 1993). The topological structures ${ }^{1}$ defined on the sets of individuals and properties can then be used to define non-modal constraints on the mappings thereby characterizing the "really possible" combinations. In particular I want to show the following:

1. The mapping approach avoids the well-known incompatiblity problems (cf. Bigelow 1988) unrestricted combinatorial accounts are notoriously plagued with. ${ }^{2}$

\footnotetext{
${ }^{1}$ Topological structures are not the only ones that might be used for this purpose. In (Mormann 1992) lattice structures have been used for similar purposes. As will turn out from the following considerations, quite a variety of mathematical structures may have a bearing on combinatorial theories of possibility. Hence, the topological combinatorialism advocated in this paper can be considered as a special case of a more general structural combinatorialism.

The contention that structure has a bearing on modality is, of course, not new: it may be traced back to Wittgenstein. In Tractatus (6.3751) he pointed out that there cannot be worlds containing particles with different velocities at the same time because this is excluded by the very structure of the physical state space which is used to define physically possible worlds. However, in the modern accounts of combinatorial possibility, the concept of structure has not played the crucial role it deserves, or so I want to argue.

${ }^{2}$ At least at first view, unrestricted combinatorialism has to allow combinatorial worlds as possible in which an individual $\mathrm{i}$ is both red and green (all over). This is absurd (cf.
} 
2. Topological constraints on combinatorial worlds offer structural criteria of distinguishing between possible and impossible combinations.

3. Topological combinatorialism is compatible with atomistic as well as with non-atomistic ontologies.

The outline of this paper is as follows: in section 2 we recall the basics of the combinatorial account of possibility and cast it in the framework of a mapping account which enables us to consider combinatorial worlds as mappings. In section 3 we use topological concepts for the introduction of complex individuals and properties. The resulting complex states of affairs are dealt with in section 4 . They lead to topologically motivated distinctions between possible and impossible combinations. We propose two different kinds of combinatorial worlds that may be dubbed the geometric and the algebraic worlds. The former are committed to an atomistic ontology, the latter not. Nevertheless they turn out to be essentially equivalent. In section 5 we elucidate the positions of logical atomism and monism with the aid of topological separation axioms. We conclude with some remarks on how the general format of a structural combinatorialism may look like in section 6 . The aim of this paper is not so much to argue that one particular version of topological combinatorialism scores best. Rather I want to show that topological concepts may help solve some problems of the combinatorial theory of possibility. More generally, topology might be useful for the elucidation of metaphysical concepts such as logical atomism and monism. ${ }^{3}$

\section{Combinatorial possibility and world mappings}

Let us start with a version of combinatorialism put forward by Armstrong (1989) and others (cf. Armstrong 1989, Bigelow 1988). The basic building blocks of the combinatorial worlds are (atomic) individuals and (atomic)

Bigelow (1988:43). In order to avoid these kinds of incompatibility problems, a friend of the unrestricted combinatorial account has to show that incompatible properties such as red and green are not basic properties. This might be difficult. Moreover, if this strategy is successful, a serious shortage of properties might arise.

${ }^{3}$ A succinct introduction to topology can be found in Davey/Priestley (1990). A fuller account is given in Vickers (1989), Grosholz (1985) discusses the interrelations between logic and topology, in particular the Stone Representation Theorem, from a philosophical point of view. Her paper also contains a useful glossary of topological and lattice theoretical terms. Actually, the topological considerations employed in this paper are quite elementary. We need not much more than the concepts of open and closed sets, and the the concept of continuity. 
properties. The set of individuals is denoted by $I$ and the set of properties by $Q$. A state of affairs $\langle\imath, p\rangle$ is an ordered pair of an individual $\imath$ and a property $p$ to be interpreted as the individual $\imath$ 's having property $p$ or the property $p$ 's being instantiated by $\imath$. The elements $\langle\imath, p\rangle \in \mathrm{J}$ are called the states of affairs of the world J. A combinatorial world is defined as a totality of states of affairs, i.e. as a relation $\mathrm{J} \subseteq I \times Q$.

Without loss of generality we may assume that the elements of $Q$ are incompatible to each other, i.e. in a combinatorial world $J$ each individual has only one property. This is seen as follows: Let $\mathrm{P}$ denote the power set of $Q$. A combinatorial world $\mathrm{J} \subseteq I \times Q$ is equivalent ${ }^{4}$ to a mapping

$$
\mathrm{f}_{\mathrm{J}}: I \rightarrow \mathrm{P}
$$

where $\mathrm{f}_{\mathrm{J}}(\imath):=\{p \mid\langle\imath, p\rangle \in \mathrm{J}\}$. In other words, $\mathrm{f}_{\mathrm{J}}$ is a relation $\mathrm{f}_{\mathrm{J}} \subseteq I \times \mathrm{P}$. Thus, a combinatorial world can be conceived of as a mapping from the set $I$ of individuals to the set $\mathrm{P}$ of incompatible properties. ${ }^{5}$ The combinatorial account based on $(*)$ will be called a mapping account of possibility. To have a not too far-fetched example of $(*)$ take I to be a set of space-time points and $\mathrm{P}$ a high-dimensional state space whose dimensions comprise mass, charge, spin and the like. What is going on at a space-time point $\imath$ is completely characterized by the value $f_{J}(\imath)$. In the world $f_{J}$ each individual occupies exactly one position in the state space P (cf. Skyrms 1993)). Another example is the following: Suppose $\mathrm{P}$ is some colour space, e.g. the colour circle (cf. Gärdenfors (1991)), and $I$ a set of individuals, each of them having exactly one colour. Then a possible world $\mathrm{f}: I \rightarrow \mathrm{P}$ is a colour distribution ${ }^{6}$. These examples show that the codomain $\mathrm{P}$ of a possible world mapping $\mathrm{f}: I \rightarrow \mathrm{P}$ need not be a power set. It may be a manifold or a vectorspace. The essential point is that $\mathrm{P}$ is a set of incompatible properties. This enables the mapping account even to cope with the second problem of combinatorialism, to wit, to distinguish between possible and impossible combinations. To see this just start over again with the original definition of a combinatorial world as a relation $\mathrm{J} \subseteq I \times \mathrm{P}$ retaining the assumption that $\mathrm{P}$ is a set of incompatible properties. Then we may distinguish between possible and impossible combinations as follows:

\footnotetext{
${ }^{4}$ That is to say, $J$ can be reconstructed from $f_{\mathrm{J}}$ and vice versa.

5 The incompatibility of the elements of $\mathrm{P}$ amounts to the trivial fact that in a world $\mathrm{J}$ an individual $\iota$ has the properties it has and no others.

${ }^{6}$ A detailed discussion of the topological and geometric structure of colour spaces can be found in Gärdenfors (1990) and Mormann (1993), concerning the problem of red/green incompatibility, see especially p. 86.
} 
Definition 1. Let $I$ be a set of individuals, $\mathrm{P}$ be a set of incompatible properties. A relation $\mathrm{J} \subseteq I \times \mathrm{P}$ is a possible combination iff it is a function from $I$ to $P{ }^{7}$ If $\mathrm{J}$ does not satisfy these requirements it is called an impossible combination. ${ }^{8}$ The logical space of all possible worlds is the set $\operatorname{Hom}(I, \mathrm{P})^{9}$ of all functions with domain $I$ and range $P$.

Let us pause for a moment and take stock what we have achieved so far. The distinction between possible and impossible combinations is achieved by imposing structural constraints on the combinatorial relation $J$ and the set P of properties. Similarly, the circumvention of incompatibility problems depends on the structural constraints imposed on P. For these achievements we have to pay a price, or so it seems: we have to rely on structural constraints imposed on the world mappings. Thus, structural constraints serve as a surrogate for modality. Taking into account structure should not, however, be seen as a vice but as a virtue. It enables us to flesh out the somewhat anemic set-theoretical combinatorialism according to which the world is just a heap of atomic states of affairs. Combinatorial worlds based on state spaces show that this is an oversimplification it is structure what really matters. I contend that structural considerations must play a crucial role in any viable combinatorial theories of possibility. The assertion that the ensembles of individuals and properties are structures spaces rather than unstructured sets is not a philosophers' idle invention. Science itself teaches us that the sets of individuals and properties are carry a lot of complex structures. For instance, if we chose space-time points as the world's atomic individuals we take them as elements of a highly structured space-time manifold. Thus, it is a legitimate aim of a philosophical theory of possibility to elucidate the essentials of those structural "scientific" accounts of possibility. The main thesis of this paper is that topological structures play an essential role in this endeavour.

${ }^{7}$ A relation $\mathrm{J} \subseteq I \times \mathrm{P}$ is a function with domain $I$ and codomain $\mathrm{P}$ iff it satisfies to the following two structural constraints: (i) $\forall \imath \in I \exists p \in \mathrm{P}\langle\imath, p\rangle \in \mathrm{J}$,

(ii) $\forall \imath \in I \forall p, p^{\prime} \in \mathrm{P}\left(\langle\imath, p\rangle \in \mathrm{J} \&\left\langle\imath, p^{\prime}\right\rangle \in \mathrm{J} \Rightarrow p=p^{\prime}\right)$.

${ }^{8}$ An impossible world $\mathrm{J}$ in the sense of Definition 1 contains states of affairs $\langle\imath, p\rangle$ and $\left\langle\imath, p^{\prime}\right\rangle$ where $p$ and $p^{\prime}$ are incompatible to each other, and/or it has individuals that have no properties at all. Each of these options qualifies J as an impossible world.

9 This denotation is borrowed from category theory (cf. Mac Lane/Moerdijk 1992). Generally ' $\operatorname{Hom}(I, \mathrm{P})$ ' is to be read as "the class of homomorphism from $I$ to P". In the case of Definition 1 the homomorphisms are just the set theoretical functions from $I$ to $P$. The usefulness of this notation will turn out in the following. 


\section{Complex individuals and topological structures}

Even if there are atomic individuals and properties, and even if we had suceeded to find out what they are, in most considerations we would still like to talk about complex individuals and properties. Complex individuals and properties are built up from atomic ones. Obviously, structures defined on the set of atomic individuals and properties should play some role in the constitution of complex individuals and properties. More precisely, they should restrict the realm of those complex entities. Not just any contrived set of atomic individuals should count as a decent complex individual. There are various options of how structure may play a role in the constitution of complex individuals and properties. I don't want deal with the problem which is best. I just mention two of them, called the Heyting approach and the Borel approach which seem to be typical. For this purpose let us recall the following basic topological definitions:

Definition 2. A topological space $\langle X, \mathscr{O}(X)\rangle$ is a set $X$ together with a collection $\mathscr{O}(X)$ of subsets of $X$ such that $\mathscr{O}(X)$ contains the empty set $\emptyset$, $X$ itself, all finite intersections, and arbitrary unions of members of $\mathscr{O}(X)$. $\mathscr{O}(X)$ is called the Heyting algebra of open subsets of the topological space $\langle X, \mathscr{O}(X)\rangle .^{10}$

A subset $Y$ of $X$ is called a closed subset if it is the complement of an open subset of $\langle X, \mathscr{O}(X)\rangle$.

Intuitively, if $x \in U(x) \in \mathscr{O}(X)$, the open set $U(x)$ is to be understood as a neighborhood of $x$ which comprises the elements of $X$ that are near or similar to $x$ in a sense specified by $U$. According to another interpretation the open neighborhoods might be thought of as predicates of the points of $X$ (cf. Vickers 1989).

Prominent examples of topological spaces are metrical spaces:

Definition 3. Let $X$ be a metric space, i.e. a set endowed with a real-valued function $d: X \times X \rightarrow \mathbb{R}$ which satisfies the familiar metrical axioms. For $x \in X$ and $\varepsilon>0$ the ball $U(x, \varepsilon):=\{y \mid d(x, y)<\varepsilon\}$ is to be defined as an open set. By arbitrary union and finite intersection the open balls generate a topology $\mathscr{O}(X)$ called the metric topology on $X$.

Perhaps the most familiar metric spaces are the Euclidean plane and its offsprings, e.g. the real line and the unit interval $[0,1]$. Metrical spaces are,

${ }^{10}$ If there is no danger of confusion $\langle X, \mathscr{O}(X)\rangle$ is abbreviated by $X$. 
however, in no way, the only examples of topological spaces. The following two topological structures exist on any set $X$ and will play an important role in the following sections:

Examples. Let $X$ be any set and $\mathscr{P}(X)$ its power set. On $X$ we have the following two extreme topologies: (i) $\mathscr{O}(X)=\mathscr{P}(X)$. This topology is called the discrete topology. (ii) The indiscrete topology defined by $\mathscr{O}(X)=\{\emptyset, X\}$.

If $X$ is endowed with the discrete topology the elements of $X$ are completely isolated from each other, i.e. each $x \in X$ has an open neighborhood, to wit the singleton $\{x\}$, which contains no other elements except $x$. Intuitively, the discrete topology renders $X$ an extremely unconnected and scattered structure. According to the indiscrete topology, there is no open neighborhood which separates different elements of $X$. Intuitively, the indiscrete topology renders $X$ an extremely connected structure in which no element can be separated from any other element.

Topological spaces abound. I dare say that all sets to be met in nature actually are topological spaces. That is to say, sets come into being only when we forget about the topological structures of topological spaces. As a first application of the topological structures one should mention that they can be used to topologically single out "nice" sets in favour of "wild" ones (cf. Gärdenfors 1991, Mormann 1993). For example, open or closed sets are more natural than those that are neither open nor closed. Indeed, starting with the set $\mathscr{O}(X)$ of open sets a full hierarchy of topologically more and more complicated sets can be defined (cf. Grosholz 1985). In the following I propose to use the first two or three levels of this hierarchy for defining complex individuals and properties:

Definition 4. Let $\langle I, \mathscr{O}(I)\rangle$ be a topological space. The elements of $I$ are to be interpreted as the atomic individuals.

(i) According to the Heyting approach, the set $\mathscr{O}(I)$ is to be taken as the set of complex individuals.

(ii) The algebra $\mathscr{B}(I)$ of Borel sets is generated by $\mathscr{O}(I)$ using the Boolean operations of complement, countable union, and intersection. According to the Borel approach, $\mathscr{B}(I)$ is to be taken as the set of complex individuals.

(iii) Analogously, $\mathscr{O}(\mathrm{P})$ and $\mathscr{B}(\mathrm{P})$ define sets of complex properties.

The usefulness of topological structures, however, is not exhausted by the definition of the topological hierarchy. Rather, the raison d'être of topological 
structures is that they provide an explication of the intuitive concept of continuity and related concepts. Among the many equivalent definitions of continuity we choose the following:

Definition 5. Let $X$ and $Y$ be topological spaces and $f: X \rightarrow Y$ a mapping. The power sets of $X$ and $Y$ are denoted by $\mathscr{P}(X)$ and $\mathscr{P}(Y)$, respectively. The map $f$ induces a map $f^{-1}: \mathscr{P}(Y) \rightarrow \mathscr{P}(X)$ defined by $f^{-1}(A):=$ $\{x \mid f(x) \in A\}, A \in \mathscr{P}(Y) . f$ is said to be continuous iff $f^{-1}$ maps open sets of $Y$ onto open sets of $X$, i.e. iff $f^{-1}$ can be restricted to a map $f^{-1}: \mathscr{O}(Y) \rightarrow \mathscr{O}(X)$

At this stage the general strategy of the topological mapping account to a combinatorial theory of possibility should be clear: first the sets of atomic individuals and properties are endowed with convenient topological structures. What is to be countenanced as convenient largely is an empirical question. The examples of physical state spaces and colour spaces give us some hints. Then the topological structures are used to define structural constraints which are imposed on "really possible" world mappings. This may be done in various ways, the most straightforward one is to stipulate possible worlds as continuous mappings.

As a first example how this may be done let us consider once again the combinatorial worlds $\mathrm{f}_{\mathrm{J}}: I \rightarrow \mathrm{P}$ as defined in $(*)$. Natural topological structures on $I$ and $\mathrm{P}$ may be defined by defining partial orders on $I$ and $\mathrm{P}$ as follows:

Definition 6. Let $\mathrm{J} \subseteq I \times Q$ be a combinatorial world.

(i) A partial order on $I$ is defined by:

$$
\imath \leq \jmath \stackrel{\mathrm{df}}{\Longleftrightarrow}
$$

(ii) A partial order on $\mathrm{P}=\mathscr{P}(Q)$ is defined by set theoretical inclusion. A topological structure $\mathscr{O}(I)$ on $I$ is defined as follows:

$$
K \in \mathscr{O}(I) \stackrel{\mathrm{df}}{\Longleftrightarrow}\{\jmath \mid \exists \imath \in K \quad \imath \leq \jmath\}=K .
$$

(iii) A topological structure $\mathscr{O}(\mathrm{P})$ on $\mathrm{P}$ is defined by

$$
\mathrm{L} \in \mathscr{O}(\mathrm{P}) \stackrel{\mathrm{df}}{\Longleftrightarrow}\{\mathrm{f} \mid \exists \mathrm{h} \in \mathrm{L} \mathrm{h} \subseteq \mathrm{f}\}=\mathrm{L} .
$$


As is well-known a map $\mathrm{f}_{\mathrm{J}}: I \rightarrow \mathrm{P}$ is continuous with respect to the topologies defined above if and only if it is order-preserving in the following sense:

$$
\forall \imath, \jmath \in I(\imath \leq \jmath \Rightarrow \mathrm{f}(\imath) \subseteq \mathrm{f}(\jmath)) .
$$

The intuitive meaning of those orders on $I$ is the following: elements $\imath$ and $k$ for which a $q$ with $\langle\imath, q\rangle$ and $(k, q)$ exist may be characterized as neighbors in J. The relation $\imath \leq \jmath$ holds iff all neighbors of $\imath$ are neighbors of $\jmath$ (the reverse may not be the case).

The set $f_{J}(\imath)$ is to be considered as the set of properties the individual $\imath$ has in the combinatorial world J. Hence, the world map $f_{J}$ is order-preserving iff an individual $\jmath$ that has more neighbors in $J$ than an individual $\imath$ has at least all the properties $\imath$ has. This seems to be a quite natural requirement. However, as is easily seen not all relations $\mathrm{J} \subseteq I \times Q$ do satisfy it. Thus, continuity of the world map $f_{J}$ happens to be an effective constraint to distinguish between "really possible" worlds and others that do not enjoy this property.

Of course, the topological approach of Definition 6 may be generalized: we no longer stick to the special order topologies of Definition 6 but generalize to other convenient topological structures. In particular, we do no longer assume that $\mathrm{P}$ has the structure of a power set, rather we are content to stipulate that it is a topological space whose elements are to be interpreted as mutually incompatible properties.

\section{Topologically possible combinatorial worlds}

Complex individuals and complex properties remain useless as long as we do not explain how they combine to form complex states of affairs. The definition of complex facts will enable us to identify the topological constraints that distinguish between possible and impossible combinations. The general result will be that only those worlds are regarded as possible whose complex individuals behave decenctly, i.e. have complex properties. This is to be spelt out as follows: Let $\mathrm{f}: I \rightarrow \mathrm{P}$ be a possible world in the sense of $(*)$. In this world the atomic individual $\imath$ has the atomic property $f(\imath)$. It is tempting to stipulate that in the world $f$ the complex (Borel or Heyting) individual $K \subseteq I$ has the property $\mathrm{f}(K)$. However, if there is no constraint on $f$ the "property" $f(K)$ might not be a Borel or Heyting property. As is mathematically well-known, this is almost always the case: thus, according to $(*)$, in most possible worlds most complex individuals would not have 
any properties at all. This does not sound plausible. In a world, an individual should have properties. Otherwise it would exist in that world but in an indeterminate and unspecified way (cf. Armstrong 1989:43). One might conclude that complex individuals and properties do not to fit the mapping account of combinatorialism. The combinatorial approach seems to be incompatible with complex individuals, properties and states of affairs. This conclusion, however, would be drawn too hastily. The situation is not hopeless. The necessary revision of $(*)$ will show the real virtues of a topological combinatorialism.

There are several strategies to avoid the shortcomings of the naive definition $(*) .{ }^{11}$ I want to pursue two of them. They may be dubbed the geometric and the algebraic account. Both rely on the plot of restricting the world mappings by suitable structural (geometric or algebraic) constraints. The geometric account relies on structural constraints defined for atomic individuals and properties, the algebraic not. Let us start with the following geometric account:

Definition 7. (Geometric worlds) A possible world is a continuous function $\mathrm{f}: I \rightarrow \mathrm{P}$. An impossible world is a discontinuous function $\mathrm{f}: I \rightarrow \mathrm{P}$. The class of complex individuals is the algebra of Borel (resp. Heyting) sets of $I$, the class of complex properties is the algebra $\mathscr{A}(\mathrm{P})$ of analytic sets ${ }^{12}$ of $\mathrm{P}$. The states of affairs of $\mathrm{f}$ is the class $\{\langle K, \mathrm{f}(K)\rangle \mid K \in \mathscr{B}(I)\}$ (resp. $\{\langle K, \mathrm{f}(K)\rangle \mid K \in \mathscr{O}(I)\})$. The logical space of possible worlds is the set $\operatorname{Hom}_{C}(I, \mathrm{P})$ of continuous mappings from $I$ to $\mathrm{P}$.

To spell out the role of the continuity constraint in some more detail the intuition behind Definition 7 may be formulated as follows: Continuity ensures that in possible combinations complex individuals get decent complex

${ }^{11}$ A rather adhoc manoeuvre to save $(*)$ I will not further pursue is the following: we stipulate a possible world to be a function $\mathrm{f}: I \rightarrow \mathrm{P}$ which maps each Borel (resp. Heyting) set $K \subseteq I$ onto a Borel (resp. Heyting) set $\mathrm{f}(K) \subseteq$ P. Consequently, an impossible world is a function $f$ that happens to map a Borel (resp. Heyting) individual onto a non-Borel (resp. non-Heyting) subset of P. This plot has the drawback that in the case of Borel sets the class of functions which satisfy this criterion is mathematically not well understood. At least, I haven't been able to find any information about those function in the literature relevant to the subject. Moreover, for Heyting worlds this definition would be obviously inappropriate since it is too restrictive. For instance, a constant function mapping all individuals to one property would not count as a possible world.

12 There are continuous functions $\mathrm{f}: I \rightarrow \mathrm{P}$ such that $\mathrm{f}(K)$ is not a Borel set even if $K \subseteq I$ is Borel (cf. Jech (1978:497f)). The image $f(K)$ of a Borel set by a continuous function always belongs the algebra $\mathscr{A}(\mathrm{P})$ of analytic sets, however. 
properties. Impossible worlds are characterized by the fact that they contain complex individuals with contrived "non-properties".

The concept of geometric combinaorial worlds depends on the the strong empirical assumption that there are atomic individuals and properties which constitute the complex individuals and properties. One might wish to get rid of this strong presupposition. This can be achieved by switching from geometric to algebraic worlds. According to the algebraic account combinatorial worlds no longer have to be atomically constituted. Although the algebraic account turns out to be essentially equivalent to the geometric it conceptually differs from the latter. It is based on the observation that a continuous map $f: X \rightarrow Y$ induces a morphism between the Heyting (or Borel) algebras of $X$ and $Y$. That is to say, even if a continuous map $\mathrm{f}: I \rightarrow \mathrm{P}$ does not necessarily map a Heyting or Borel individual $K$ onto a Heyting or Borel property $f(K)$ the reverse set theoretical map $f^{-1}: 2^{\mathrm{P}} \rightarrow 2^{I}$ does. It even induces a Borel homomorphism $\mathrm{f}^{-1}: \mathscr{B}(\mathrm{P}) \rightarrow \mathscr{B}(I)$. Hence, if we forget about the set theoretical map $f$ we may define a possible world as a morphism of Borel algebras $\mathrm{h}: \mathscr{B}(\mathrm{P}) \rightarrow \mathscr{B}(I)$ (or as a morphism $\mathrm{k}: \mathscr{O}(\mathrm{P}) \rightarrow \mathscr{O}(I)$ of Heyting algebras). This move requires some further adjustments concerning the states of affairs of the possible world redefined in this manner. How they should be defined? A straightforward answer is to be recommended: Given $h$, the property $L \in \mathscr{B}(P)$ is instantiated by the (Borel or Heyting) individual $h(L)$. Hence, a state of affairs of the world $h$ should be a pair $\langle L, h(L)\rangle$. Or, to state it in a different way, in $h$ the individual $h(L)$ has the property L. However, this definition is not quite right. According to it, we lose a lot of states of affairs we probably like to retain and get into conflict with the principle that every individual in a possible world should have a property therein. This is seen as follows: assume that $\langle L, h(L)\rangle$ is a state of affairs in $h$. For a complex individual $K \subseteq h(\mathrm{~L})$ we cannot guarantee that there is a $\mathrm{L}^{\prime}$ with $\mathrm{h}\left(\mathrm{L}^{\prime}\right)=K$. Thus, the following definition suggests itself:

Definition 8. (Atomistic algebraic Borel worlds) Let $I$ and $\mathrm{P}$ be the spaces of individuals and properties, respectively. A possible world is a morphism of Borel algebras $h: \mathscr{B}(\mathrm{P}) \rightarrow \mathscr{B}(I)$. The class of states of affairs of $h$ is the class $\{\langle\mathrm{L}, K\rangle \mid \mathrm{L} \in \mathscr{B}(\mathrm{P}) \& K \in \mathscr{B}(I) \& K \subseteq \mathrm{h}(\mathrm{L})\}$. The logical space of all possible worlds is the class of Borel morphisms $\operatorname{HOM}_{\mathscr{B}}(\mathscr{B}(\mathrm{P}), \mathscr{B}(I))$ from $\mathscr{B}(\mathrm{P})$ to $\mathscr{B}(I)$. An impossible world is given by a map h: $\mathscr{B}(\mathrm{P}) \rightarrow \mathscr{B}(I)$ which fails to be a Borel morphism. ${ }^{13}$

\footnotetext{
${ }^{13}$ Quite analogously, we may define possible worlds based on the Heyting algebras $\mathscr{O}(\mathrm{P})$ and $\mathscr{O}(I)$. In this case the logical space is the set $\operatorname{Hom}_{H}(\mathscr{O}(\mathrm{P}), \mathscr{O}(I))$ of Heyting morphisms.
} 
As Skyrms (1993) has pointed out the definition of possible worlds as a Borel or Heyting algebra morphisms paves the way for a genuinely nonatomistic combinatorial theory of possibility: we forget about the underlying topological spaces $I$ and $\mathrm{P}$ defining the individuals and properties of such a world as elements of the appropriate Borel or Heyting algebras:

Definition 9. (General algebraic Borel worlds) Let $\mathscr{B}_{I}$ and $\mathscr{B}_{\mathrm{P}}$ be Borel algebras to be interpreted as the classes of complex individuals and properties, respectively. A possible world is a Borel algebra morphism h: $\mathscr{B}_{\mathrm{P}} \rightarrow \mathscr{B}_{I}$. The states of affairs of the world $h$ are defined as in Definition 8. The logical space of all possible worlds is the set $\operatorname{HOM}\left(\mathscr{B}_{\mathrm{P}}, \mathscr{B}_{I}\right)$ of Borel morphisms between $\mathscr{B}_{\mathrm{P}}$ and $\mathscr{B}_{I}$. An impossible world is a map $k: \mathscr{B}_{\mathrm{P}} \rightarrow \mathscr{B}_{I}$ that is not a Borel morphism. ${ }^{14}$

The main advantage of these algebraic concepts of combinatorial worlds is that we we are no longer restricted to worlds constitutited by atomic individuals and properties. As is well known, Borel and Heyting algebras generally have no atoms. ${ }^{15}$ Thus, the general algebraic account of Heyting or Borel worlds shows that the combinatorial approach need not subscribe to atomism. This should be considered as a definitive advantage. That the ultimate structure of the world is atomistic is to be considered as an empirical claim a metaphysical theory such as the combinatorial account to possibility should not depend on.

To sum up we may say that the topological mapping account offers two types of combinatorial worlds: on the one hand we have the geometric account which conceptualizes possible combinatorial worlds as continuous mappings; on the other hand we have the algebraic account which conceptualizes possible combinatorial worlds as morphisms of algebras. The geometric combinatorial worlds are constituted by atomic states pf affairs constituted by atomic individuals and properties. Generally, this does not hold for algebraic worlds: Borel and Heyting worlds may be genuinely non-atomistic, i.e. they may contain no atomic states of affairs at all.

How these two types of combinatorial worlds are related? The answer is as smooth as can be. Using the Stone Representation theorem, Skyrms has pointed out that the geometric and algebraic Borel worlds are essentially

\footnotetext{
14 An analogous definition might be given for worlds based on Heyting algebras of complex individuals and properties.

${ }^{15}$ A common example is provided by the Heyting algebra $\mathscr{O}([0,1])$ of the unit interval $[0,1]$.
} 
equivalent. Stone's theorem asserts (see Vickers (1989), Th. 9.5.4) that for any Boolean algebra $B$ there is a topological space $\operatorname{St}(B)$ such that $B$ is isomorphic to the field of all subsets of $\operatorname{St}(B)$ that are closed and open with respect to the topology of $\operatorname{St}(B)$. Boolean homomorphisms from $\mathscr{B}_{\mathrm{P}}$ to $\mathscr{B}_{I}$ are in 1-1-correspondence with the continuous maps from $\operatorname{St}\left(\mathscr{B}_{I}\right)$ to $\operatorname{St}\left(\mathscr{B}_{\mathrm{P}}\right)$. Hence, if we are ready to inflate our ontology from the Borel algebras of complex properties and individuals to their Stone spaces we arrive at the geometric combinatorial worlds constituted by atomic individuals and properties. Vice versa, a geometric world $\mathrm{f}: I \rightarrow \mathrm{P}$ gives rise to an algebraic world $\mathrm{f}^{-1}: \mathscr{B}(\mathrm{P}) \rightarrow \mathscr{B}(I)$.

Similarly, under some mild assumptions, the algebraic approach for Heyting worlds can be shown to be equivalent to the gemetric approach ${ }^{16}$, i.e., a geometric Heyting world gives rise to an algebraic Heyting world and vice versa. Thus, the non-atomistic algebraic versions of the combinatorial approach based on the algebras of Borel or Heyting sets can be cast into the same framework of topological combinatorialism as the atomistic geometric ones based on continuous mappings.

\section{A topological elucidation of atomism and monism}

The ideas of logical atomism may be traced back to Wittgenstein's Tractatus and Russell's Philosophy of Logical Atomism. Both were opposed to the monism of the British idealists of that time who held that reality is one single thing, not many - even if our limited cognizing capacity forces us to think of it as it were composed of many independent separate things (cf. Bradley 1992:5).

Taking states of affairs as the ultimate constituents of the world, I consider take atomism to be the doctrine that the states of affairs into which the world divides are all independent of each other. Thus, atomism subscribes to a principle of independence according to which an individual's $\imath$ having a property $p$ is independent of an individual's $\jmath$ having the property $q$. The principle of independence is denied by monistic doctrines. According to them, single states of affairs are only abstractions, and the independence principle is radically wrongheaded. If one state of affairs is properly understood it determines the whole world, i.e., all other states of affairs. These

16 A Heyting algebra $H$ gives rise to a topological space $\mathrm{T}(H)$ in such a way that a Heyting morphism $h: H \rightarrow H^{\prime}$ induces a continuous map $\mathrm{T}(h): \mathrm{T}\left(H^{\prime}\right) \rightarrow \mathrm{T}(H)$. Under reasonable conditions, i.e. if $H$ is spatial (cf. Mac Lane/Moerdijk (1992:478) the Heyting algebra $\mathscr{O}(\mathrm{T}(H))$ of open sets of $\mathrm{T}(H)$ is just $H$ again. 
vague claims may be elucidated with the help of topological separation axioms, or so I want to argue. Let us start within the naive mapping account of $(*)$. In this framework the independence principle for atomic individuals may be formulated as follows:

5.1. Atomic independence principle. Given any two different atomic individuals $\imath$ and $\jmath$ and any two properties $p$ and $q$ there is a possible world mapping $\mathrm{f}: I \rightarrow P$ with $\mathrm{f}(\imath)=p$ and $\mathrm{f}(\jmath)=q$.

Because no constraints are imposed on $\mathrm{f}$ we stipulate $\mathrm{f}(\imath)=p$ and $\mathrm{f}(\jmath)=q$ and define it as we like on the rest of $I$. This, of course, is not too exciting. A somewhat more interesting problem arises if we put the topological structures of $I$ and $\mathrm{P}$ to work. That means, we define a possible world as a continuous function $\mathrm{f}: I \rightarrow \mathrm{P}$. Is it still true that there is a world $\mathrm{f}$ with $\mathrm{f}(\imath)=p$ and $\mathrm{f}(\jmath)=q$ ? The general answer is NO. We have to assume that $I$ is at least an Urysohn space. ${ }^{17}$ This is not a particularly strong restriction, all "reasonable" sets of individuals, e.g. spacetime manifolds, are Urysohn spaces.

What about independence principles for disjoint complex individuals (cf. Armstrong 1989:69/70)? A quite strong (and naive) independence principle for complex individuals reads as follows:

5.2. General independence principle. Given any two disjoint individuals $\imath$ and $\jmath$ and any two properties $p$ and $q$ there is a possible combinatorial world $\mathrm{f}: I \rightarrow \mathrm{P}$ in which $\imath$ has property $p$ and $\jmath$ has property $q$, i.e. $\mathrm{f}(\imath)=p$ and $\mathrm{f}(\jmath)=q$.

If we put no restrictions on the world mapping $f, 5.2$ is valid for trivial reasons. More interesting is the case of continuous worlds. Topology offers a well assorted box of different possibilities. I don't want to discuss them all in detail, and I mention just one:

5.3. Independence principle for closed individuals. Let $\imath$ and $\jmath$ be two disjoint closed individuals and $p$ and $q$ two properties. There is a continuous

\footnotetext{
${ }^{17} X$ is an Urysohn space iff for any two different points $a, b \in X$ there is a continuous function $f: X \rightarrow[0,1]$ such that $f(a)=0$ and $f(b)=1$. The Urysohn condition is slightly stronger than the more familiar Hausdorff separation axiom stating that any two different points $a$ and $b$ can be separated by disjoint open sets $O(a)$ and $O(b)$ containing $a$ and $b$ respectively (cf. Seebach/Steen 1978, section 2).
} 
world $\mathrm{f}: I \rightarrow \mathrm{P}$ with $\mathrm{f}(\imath)=p$ and $\mathrm{f}(\jmath)=q$ iff $I$ is a normal topological space. $^{18}$

As is easily seen, an independence principle for non-closed disjoint individuals does not hold for continuous possible worlds: Take $I$ to be the unit interval $[0,1]$ endowed with the standard topology. Let $K$ denote the complex Borel individual of rational numbers. Then, in a continuous world $\mathrm{f}: I \rightarrow \mathrm{P}$ the property $\mathrm{f}(K)$ of $K$ completely determines the properties of all other individuals. This case can easily be generalized to differentiable manifolds. Thus, if we take combinatorial worlds defined by physical state spaces as a guideline an unrestricted principle of indenpendence for complex individuals cannot be maintained anymore. In other words, scientifically possible combinatorial worlds are less atomistic and more monistic than an extreme Russellian atomism might be prepared to accept. It should be noted that the fact that a general independence principle for disjoint complex individuals does not hold depends on the fact that we maintained a conservative stance with respect to the topological structures defined on $I$ and P. By brute force, i.e. if we are prepared to drastically change the topological structure of $I$ or $\mathrm{P}$ we arrive at radically atomistic and monistic continuous combinatorial worlds as is shown by the following examples:

5.4. Examples. (i) Let the set $\mathrm{P}$ of properties be endowed with the discrete topology. Then a continuous possible world $\mathrm{f}: I \rightarrow \mathrm{P}$ is completely determined by the property $f(\imath)$ of some individual $\imath$.

(ii) Let the set $\mathrm{P}$ of properties be endowed with the indiscrete topology. Then, for any disjoint individuals an unrestricted independence principle holds with respect to worlds $\mathrm{f}: I \rightarrow \mathrm{P}$.

Proof. (i) Chose any $\imath \in I$. Since $f$ is continuous, the set $f^{-1}(f(\imath))$ is open. Since $I$ is connected this amounta to the requirement that $f$ is constant, i.e. $\mathrm{f}(\jmath)=\mathrm{f}(\imath)$ for all $\jmath \in I$.

(ii) If $\mathrm{P}$ is endowed with the indiscrete topology, the condition of continuity is empty, i.e. just any $f$ is continuous. Thus, an unrestricted independence principle holds.

${ }^{18}$ A topological space $X$ is called normal iff it satisfies the following two conditions (cf. Steen/Seebach 1978:11):

(1) If $a, b \in X$ there are open set $O(a)$ and $O(b)$ containing $a$ and $b$ respectively such that $b \notin O(a)$ and $a \notin O(b)$;

(2) If $A$ and $B$ are disjoint closed sets in $X$, there are disjoint open sets $O(A)$ and $O(B)$ containing $A$ and $B$ respectively. Normal spaces are well-known to satsify the Urysohn condition. 
Hence, the world of 5.4(i) is a monist world in the sense of Bradley: If you really understood any one state of affairs, i.e. if you were able to determine its properties you would understand everything, i.e. you would understand all states of affairs (cf. Russell (1918:204)), while the world 5.4(ii) is an atomistic world in the strictest possible sense: even if you knew quite a lot of many states of affairs you would still be completely ignorant about the rest. In such an atomic world no kind of induction or prediction would make any sense. As can be seen from this example, the stances of extreme monism and atomism may be topologically characterized as the two ends of a spectrum of more plausible intermediate positions. Thus, topology may be a useful device for elucidating the sweeping and vague theses of those broad metaphysical claims in more precise way.

\section{Concluding remarks}

In this final section I want to outline the general format of a structural combinatorialism as it is recommended by the topological accounts developed in the previous sections. The general format of a combinatorial theory of possibility put forward in this paper can be displayed best borrowing a piece of notation from category theory (Mac Lane/Moerdijk (1992:11f)). Let $I$ and $\mathrm{P}$ be two structures of an appropriate type (for example topological spaces or Boolean algebras), to be interpreted as individuals and properties respectively. The class of structure preserving morphisms from $I$ to $\mathrm{P}$ is denoted by $\operatorname{Hom}_{C}(I, \mathrm{P})$. Here $C$ symbolizes the type (actually the category) of structure preserving constraints put on the admissible morphisms $\mathrm{f}: I \rightarrow$ P. Possible worlds are structure preserving mappings.

The set $\operatorname{Hom}_{C}(I, \mathrm{P})$ is to be considered as the logical space of possible worlds of the combinatorial account. If we forget about the structural constraints of $C$ we get a larger class of morphism $\operatorname{Hom}(I, \mathrm{P})$ which still gives rise to combinatorial worlds. Combinatorial worlds $\mathrm{f}: I \rightarrow \mathrm{P}$ belonging to $\operatorname{Hom}(I, \mathrm{P})$ but not to $\operatorname{Hom}_{C}(I, \mathrm{P})$, are to be considered as impossible worlds. Or, if one does not want to draw a sharp line between the possible and the impossible, those worlds might be characterized as far-fetched or absurd worlds.

Thus, the distinction between possible and impossible worlds essentially depends on the structural constraints $C$. For the sake of definiteness, let us concentrate on the case where $C$ can be interpreted as the constraint of continuity. The continuity of a world map $\mathrm{f}: I \rightarrow \mathrm{P}$ depends on the topological structures defined on $I$ and P. These structures are to be conceived 
of as higher order states of affairs (cf. Armstrong (1989:87f)). A world is, so to speak, a topologically structured totality of states of affairs, or, to express somewhat more generally, it is a structural gestalt (cf. Mormann 1994). Hence, it is not completely described by a world function $f: I \rightarrow P$. Although $f$ uniquely determines the totality of first-order states of affairs the higher order states of affairs are missing. Thus, in a topologically refined combinatorial approach there can be distinct worlds which share all the same first order states of affairs but differ with respect to higher order states of affairs. ${ }^{19}$ The introduction of higher-order states of affairs may rise some suspicions among nominalistically-minded philosophers. But I think the combinatorial approach is justified to do this step. It must even do it. Science has gathered ample evidence that the actual world cannot be described in the framework of a strict first-order nominalism. The combinatorial approach to possibility should accommodate to this fact admitting topological and other higher order structural states of affairs. ${ }^{20}$

\section{References}

Armstrong, D. M., A Combinatorial Theory of Possibility, Cambridge, Cambridge University Press, 1989.

Bigelow, J. C., "Real possibilities", Philosophical Studies 53 (1988), 37-64.

Bradley, R., The Nature of All Being. A Study of Wittgenstein's Modal Atomism, Oxford, Oxford University Press, 1992.

Davey, B. A., and H. A. Priestley, Introduction to Lattices and Order, Cambridge, Cambridge University Press, 1990.

Gärdenfors, P., "Induction, conceptual spaces, and AI", Philosophy Of Science 57 (1990), 78-95.

Grosholz, E., "Two episodes in the unification of logic and topology", The British Journal Of Philosophy Of Science 36 (1985), 147-57.

Jech, Th., Set Theory, New York, Academic Press, 1978.

Loux, M. J., (ed.), The possible and the actual, Readings in the Metaphysics of Modality, Ithaca, Cornell University Press, 1979.

\footnotetext{
${ }^{19}$ For this reason topological combinatorialism is not compatible with Skyrms's "Tractarian Nominalism" where only first order facts are admitted (cf. Armstrong (1989:87), Skyrms (1981: 202-203)).

${ }^{20}$ As Armstrong (1989:section 7) has pointed out, even a non-topological combinatorial approach must admit higher order entities already for the very definition of a combinatorial world.
} 
Lycan, W., "The trouble with possible worlds", in M. J. Loux (ed.) The Possible and the Actual, 274-316, 1979.

Mac Lane, S., and I. Moerdijk, Sheaves in Geometry and Logic, New York, Springer, 1992.

Mormann, T., "Structural accessibility and similarity of possible worlds", Journal Of Philosophical Logic 21 (1992), 149-172.

Mormann, T., "Natural predicates and topological structures of conceptual spaces", Synthese 95 (1993), 219-240.

Mormann, T., "Accessibility, kinds, and laws: a structural explication", Philosophy Of Science 61 (1994), 389-406.

Russell, B., The Philosophy of Logical Atomism, edited by D. Pears, Open Court.

Skyrms, B., "Tractarian nominalism", Philosophical Studies 40 (1981), 199-206.

Skyrms, B., "Logical atoms and combinatorial possibility", The Journal Of Philosophy 95 (1993), 219-232.

Steen, L. A., and J. A. Seebach, Counterexamples in Topology, Springer Verlag, New York, 1978.

Vickers, St., Topology via Logic, Cambridge University Press, Cambrigde, 1989.

Thomas Mormann

IPLW, Universität München

Ludwigstr. 31

D-80539 München, Germany 